Received: 8 November 2016, Accepted: 4 January 2017

Edited by: D. Gomez Dumm

Licence: Creative Commons Attribution 3.0

DOI: http://dx.doi.org/10.4279/PIP.090002

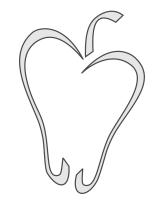

ISSN 1852-4249

\title{
An alternative derivation of the Dirac operator generating intrinsic Lagrangian local gauge invariance
}

\begin{abstract}
Brian Jonathan Wolk ${ }^{1 *}$
This paper introduces an alternative formalism for deriving the Dirac operator and equation. The use of this formalism concomitantly generates a separate operator coupled to the Dirac operator. When operating on a Clifford field, this coupled operator produces field components which are formally equivalent to the field components of Maxwell's electromagnetic field tensor. Consequently, the Lagrangian of the associated coupled field exhibits internal local gauge symmetry. The coupled field Lagrangian is seen to be equivalent to the Lagrangian of Quantum Electrodynamics.
\end{abstract}

\section{Introduction}

The Dirac equation [1] arises from a Lagrangian which lacks local gauge symmetry [2-6]. In the usual quantum field theoretic development, local gauge invariance is thus made an external condition of and on the Lagrangian [3-6]. Introduction of a vector field $A_{\mu}$ that couples to the Dirac field $\psi$ must then be introduced in order to satisfy the imposed local symmetry constraint [2-4].

More satisfactory from a theoretic standpoint would be a formalism in which derivation of the Dirac operator equation is associated with a Lagrangian exhibiting internal local gauge symmetry. Such a formalism would alleviate both the need to impose local gauge invariance as an external mandate as well as the need to invent and introduce a vector field to satisfy the constraint. Symmetry would exist $a b$ initio. This paper presents such an approach and derivation.

\footnotetext{
*E-mail: attorneywolk@gmail.com

13551 Blairstone Road, Tallahassee, FL 32301 Suite 105, USA.
}

\section{Alternative formalism}

\section{i. The standard approach}

One consequence of the standard approach [1-6, $8,20]$ in deriving the Dirac operator $\not \supset \equiv \gamma_{\alpha} \partial^{\alpha}=$ $\gamma_{0} \partial / \partial t-\gamma \cdot \nabla$, with $\gamma=\left(\gamma_{1}, \gamma_{2}, \gamma_{3}\right)$, which is related to the d'Alembertian operator $\square \equiv \partial^{\mu} \partial_{\mu}$ associated with the Klein-Gordon equation $\square \psi=-M^{2} \psi[2-$ $6,20]$, is that Clifford-Dirac elements $\left\{\gamma^{\mu}\right\}$ arise as necessary structures of the Dirac operator $\not \partial$, with the following properties $[2-6,8,10,21]$

$$
\begin{aligned}
& \gamma^{\alpha}=g^{\alpha \delta} \gamma_{\delta} ; \quad \gamma_{\mu} \gamma^{\mu}=4 \\
& \gamma^{n} \gamma^{m}=-\gamma^{m} \gamma^{n}(n \neq m) \\
& \left(\gamma^{n}\right)^{2}=-1 ; \quad\left(\gamma^{0}\right)^{2}=1 \\
& \left(\gamma^{\mu}\right)^{\dagger}=\gamma^{0} \gamma^{\mu} \gamma^{0} \quad\left(\gamma^{n}\right)^{\dagger}=-\gamma^{n} \\
& \square=\not \not^{2} .
\end{aligned}
$$

Dirac's equation $\not \partial \psi= \pm i M \psi$ follows for a fermionic field $\psi$ such as the electron [2-6,21]. 
Papers in Physics, vol. 9, ART. 090002 (2017) / B. Wolk

\section{ii. An alternate formalism}

Two conditions are set forth for developing an alternative formalism for deriving an operator, call it $\mathcal{O}$, which operates on the wave function $\psi$ for the subject fermionic particle and generates the equation governing its evolution.

The first condition is that since the wave function $\psi$ is a spinor, the Clifford elements must act, if at all, as operators on it $[8,13,20]$. Therefore, the applicable operator $\mathcal{O}$ should contain Clifford algebra elements.

The second condition is that $\square$ should be derivable from $\mathcal{O}[2,4,6] ;^{1}$ there must exist a mapping $\digamma: \mathcal{O} \rightarrow \square$, and thus the governing equation itself must satisfy ${ }^{2}$

$$
\digamma(\mathcal{O}) \psi=\digamma( \pm i M) \psi \longrightarrow \square \psi=-M^{2} \psi
$$

To satisfy the d'Alembertian condition that $\digamma$ : $\mathcal{O} \rightarrow \square$, the mapping must make use of the partial derivative operators, and so the operator $\partial \equiv$ $(\partial / \partial t, \nabla)$ is defined. To meet the Clifford condition that $\mathcal{O}$ contains Clifford elements, the operator $\eta \equiv\left(\gamma_{0}, \gamma\right)$ is put. Written explicitly, these fundamental operators are

$$
\partial=\partial / \partial t+i \partial / \partial x+j \partial / \partial y+k \partial / \partial z
$$

and

$$
\eta=\gamma_{0}+i \gamma_{1}+j \gamma_{2}+k \gamma_{3} .
$$

We wish to use these fundamental operators in constructing $\mathcal{O}$. To do so, use is made of the equivalence between the ring of quaternions $\mathbb{H}$ with basis $(1, i, j, k)$ and $\mathbb{R}^{4}$ - the four-dimensional vector space over the real numbers: $\{q \in \mathbb{H}: q=$ $\left.u_{0} 1+b i+c j+d k \mid u_{0}, b, c, d \in \mathbb{R}\right\}[10-12,14]$, with $i^{2}=j^{2}=k^{2}=-1$. The quaternion $q$ can then be divided into its scalar and vector portions: $\left\{q=\left(u_{0}, u\right) \mid u_{0} \in \mathbb{R}, u \in \mathbb{R}^{3}\right\}[11,12,14]$.

\footnotetext{
${ }^{1}$ A condition also imposed by Dirac. Ref. [2], p. 86.

${ }^{2}$ Putting $\digamma( \pm i M)=-M^{2}$ in Eq. (2) presumes that the form and domain of the mapping $\digamma$ is known. The more general relation would be $\digamma(f[M])=-M^{2}$, with $f[M]=$ $\pm i M$ to be subsequently deduced. But once the form of $\mathcal{O}$ is discovered, the form of $\digamma$ becomes evident, namely $\digamma=[]^{2}$, and deducing $f[M]$ becomes trivial. Additionally however, given the fact that the RHS of Eq. (2) involves a square, one can intuit the correct form for $\digamma$ in the first instance.
}

In this way, the operators given in (3) and (4) can be conceived as quaternionic operators, with the relations between the quaternionic basis elements and the Clifford elements being [11,13]

$$
1=\gamma_{0} \gamma_{0}, \quad i=\gamma_{2} \gamma_{3}, \quad j=\gamma_{3} \gamma_{1}, \quad k=\gamma_{1} \gamma_{2}
$$

The $\gamma_{\mu}$ are then the first-order, primary entities $[8,10,20]$ from which the quaternionic basis is constructed. ${ }^{3}$

To generate a new operator using the fundamental operators, the product $\eta \partial$ is taken. The product of two quaternionic operators $\mathbf{v}=\left(v_{0}, v\right)$ and $\mathbf{w}=\left(w_{0}, w\right)$ may be written as a product of their scalar and vector components in the $\mathbb{R}^{4}$ representation using the formula

$\left(v_{0}, v\right)\left(w_{0}, w\right)=\left(v_{0} w_{0}-\vec{v} \cdot \vec{w}, v_{0} \vec{w}+\vec{v} w_{0}+\vec{v} \times \vec{w}\right)$,

where $v \rightarrow \vec{v}$, and $w \rightarrow \vec{w}[10-14]$. This gives

$$
\eta \partial \equiv\left(\gamma_{0}, \gamma\right)(\partial / \partial t, \nabla)
$$

producing the operator

$$
\begin{aligned}
\eta \partial & \equiv\left([\eta \partial]_{0},[\eta \partial]_{\wedge}\right) \\
& =\left(\gamma_{0} \partial / \partial t-\gamma \cdot \nabla, \gamma_{0} \nabla+\gamma \partial / \partial t+\gamma \times \nabla\right) .
\end{aligned}
$$

The operator $\eta \partial$ is composed of two coupled operators (and thus will operate on two coupled fields). Its first component operator is

$$
[\eta \partial]_{0}=\gamma_{0} \partial / \partial t-\gamma \cdot \nabla .
$$

Setting $\digamma=[]^{2}$ gives a mapping $\digamma:[\eta \partial]_{0} \rightarrow \square$. This mapping satisfies Eq. (2) [6,20]. The operator $[\eta \partial]_{0}$ thus satisfies both the d'Alembertian and Clifford conditions. Putting $[\eta \partial]_{0}=\mathcal{O}$ and noting the obvious equivalence $\mathcal{O} \equiv \not \partial$, the Dirac operator is thus seen to be derived from the new formalism.

Given Eq. (2), we have $\mathcal{O} \psi= \pm i M \psi$ as a possible fermion field equation of motion. As any solution to $\mathcal{O} \psi= \pm i M \psi$ is also a solution to the KleinGordon equation [2,6,21], this equation is naturally postulated as governing a fermionic particle such as the electron.

\footnotetext{
${ }^{3}$ Note is made that $\eta=\gamma_{0} \gamma_{0} \gamma_{0}+\gamma_{1} \gamma_{2} \gamma_{3}+\gamma_{1} \gamma_{2} \gamma_{3}+$ $\gamma_{1} \gamma_{2} \gamma_{3}$
} 
Papers in Physics, vol. 9, ART. 090002 (2017) / B. Wolk

\section{The coupled operator}

A new operator which is coupled to $\not \partial$ is seen to arise within this formalism. This operator is the vector component of $\eta \partial$ in Eq. (8), namely

$$
[\eta \partial]_{\wedge}=\gamma_{0} \nabla+\gamma \partial / \partial t+\gamma \times \nabla .
$$

To maintain consistency with the formalism used with $\not \partial$, the operator $[\eta \partial]_{\wedge}$ is also written in the $\gamma_{\mu^{-}}$ basis. Designating this operator as $\boldsymbol{\gamma}$ we have

$$
\begin{aligned}
{[\eta \partial]_{\wedge} \rightarrow \boldsymbol{\gamma} } & =\gamma_{0}(\hat{i} \partial / \partial x+\hat{j} \partial / \partial y+\hat{k} \partial / \partial z) \\
& +\gamma_{1}(\hat{i} \partial / \partial t-\hat{j} \partial / \partial z+\hat{k} \partial / \partial y) \\
& +\gamma_{2}(\hat{i} \partial / \partial z+\hat{j} \partial / \partial t-\hat{k} \partial / \partial x) \\
& +\gamma_{3}(-\hat{i} \partial / \partial y+\hat{j} \partial / \partial x+\hat{k} \partial / \partial t) .
\end{aligned}
$$

Since the operators $(\not \partial, \boldsymbol{\lambda})$ are coupled, when $\not \partial$ operates on some field so should $\boldsymbol{\gamma}$. Inspection of Eq. (11) shows that $\boldsymbol{\gamma}$ 's operation must be of a different sort and on a different yet coupled field. To see how $\boldsymbol{\gamma}$ operates and on what, some notation is first required.

$\mathbf{A}=A_{0}(x)+A_{1}(x) \hat{i}+A_{2}(x) \hat{j}+A_{3}(x) \hat{k}$ represents a four-vector field, for which we can associate the Clifford field $A=A_{\mu} \gamma^{\mu}$, with $A_{\mu} \equiv A_{\mu}(x)$ being the field components of $\mathbf{A}$. There is thus a component-wise bijection between $A$ and $\mathbf{A}$.

A Clifford vector field is defined as $\not{\ell}=\mathbf{C}^{\mu} \gamma_{\mu}$, with each $\mathbf{C}^{\mu}$ being its own vector field. In this way, a general Clifford vector field operator is defined as $\not \subset=\triangle^{\alpha} \gamma_{\alpha}$, with each component $\triangle^{\alpha}$ being its own vector field operator.

In standard vector analysis, vector field operators operate on scalar fields [15]. Following suit, in order for a Clifford vector field operator's $(\not)$ component vector field operators $\left(\triangle^{\alpha}\right)$ to operate on the scalar fields $A^{\mu}$ of a Clifford field $A$, an operation - must be defined such that

$$
\not \subset \cdot A=\triangle^{\mu} \gamma_{\mu} \gamma^{\mu} A_{\mu}=\triangle^{\mu} A_{\mu}=\triangle^{\mu} A^{\nu} g_{\mu \nu}
$$

Using this formalism, the components $\boldsymbol{\nabla}^{\alpha}$ of $\boldsymbol{\gamma}$ are given by Eq. (11). ${ }^{4}$ Choosing a Clifford field of the general form

\footnotetext{
${ }^{4}$ For instance, $\boldsymbol{\nabla}^{0}=\nabla$.
}

$$
\Phi \equiv \gamma^{\mu} \Phi_{\mu}=\gamma^{0} \Phi_{0}+\gamma^{1} \Phi_{1}+\gamma^{2} \Phi_{2}+\gamma^{3} \Phi_{3},
$$

with $\Phi_{\mu} \equiv \Phi_{\mu}(x)$, and operating on $\Phi$ with $\boldsymbol{\gamma}$ gives

$$
\begin{aligned}
\boldsymbol{\nabla} \cdot \Phi & =(\hat{i} \partial / \partial x+\hat{j} \partial / \partial y+\hat{k} \partial / \partial z) \Phi_{0} \\
& +(\hat{i} \partial / \partial t-\hat{j} \partial / \partial z+\hat{k} \partial / \partial y) \Phi_{1} \\
& +(\hat{i} \partial / \partial z+\hat{j} \partial / \partial t-\hat{k} \partial / \partial x) \Phi_{2} \\
& +(-\hat{i} \partial / \partial y+\hat{j} \partial / \partial x+\hat{k} \partial / \partial t) \Phi_{3} .
\end{aligned}
$$

We have then the coupled field $\left(\psi, \Phi_{\mu}\right)$ through action of the operator $\eta \partial$. Unlike $\psi$, the $\Phi_{\mu}$ are not 4-element column matrices and are not spinor fields, since operating through in Eq. (14) excises the Clifford elements. Rearranging terms give the following set of six vector field components:

$$
\begin{array}{r}
\left(\partial \Phi_{0} / \partial x+\partial \Phi_{1} / \partial t\right) \hat{i} \\
\left(\partial \Phi_{0} / \partial y+\partial \Phi_{2} / \partial t\right) \hat{j} \\
\left(\partial \Phi_{0} / \partial z+\partial \Phi_{3} / \partial t\right) \hat{k} \\
\left(\partial \Phi_{2} / \partial z-\partial \Phi_{3} / \partial y\right) \hat{i} \\
\left(\partial \Phi_{3} / \partial x-\partial \Phi_{1} / \partial z\right) \hat{j} \\
\left(\partial \Phi_{1} / \partial y-\partial \Phi_{2} / \partial x\right) \hat{k}
\end{array}
$$

These equations can be identified with the components of two vector fields

$$
\mathbf{X}=-\nabla \Phi_{0}-\partial \vec{\Phi} / \partial t
$$

and

$$
\mathbf{Y}=\nabla \times \vec{\Phi}
$$

with $\vec{\Phi}=\left(\Phi_{1}, \Phi_{2}, \Phi_{3}\right)$. These equations represent the six independent components of an antisymmetric field tensor $\mathbf{H}$, which $\boldsymbol{\nabla} . \Phi$ has generated. There is thus a one-to-one and onto correspondence: $\{ \pm \boldsymbol{\gamma} \cdot \Phi \leftrightarrow \mathbf{H}\}$. Therefore, $\mathbf{H}$ can be written as the curl of the Clifford scalar field components

$$
H_{\mu \nu} \equiv \partial_{\mu} \Phi_{\nu}-\partial_{\nu} \Phi_{\mu}
$$

$\mathbf{H}$ is then formally equivalent to the electromagnetic field tensor $[6,16,19,22]$. Using the component-wise bijection stated above: $\{A \leftrightarrow \mathbf{A}\}$, 
Papers in Physics, vol. 9, ART. 090002 (2017) / B. Wolk

the components of $\Phi$ are identified with the components of the electromagnetic potential vector $\mathbf{A}$ : $A_{\mu} \equiv \Phi_{\mu}$. This being the case, $A_{\mu}{ }^{5}$ represents a massless vector field (the photon) abiding by the gauge invariance condition $[2,3,6,9,17-19,22]$

$$
A_{\mu} \longrightarrow A_{\mu}+\partial_{\mu} \lambda
$$

\section{i. The coupled locally gauge symmetric La- grangian}

The gauge invariance condition, Eq. (19), can be exploited to impose an additional constraint on the potential $A_{\mu}$, namely the Lorenz condition $\partial_{\mu} A^{\mu}=$ $0[2,6] .{ }^{6}$ With the aid of the Lorenz gauge, the Lagrangian for the field $A_{\mu}$ with source $J^{\mu}[2,6,18]$ can be written as

$$
\mathcal{L}_{A_{\mu}}=-\frac{1}{16 \pi} H^{\mu \nu} H_{\mu \nu}-\frac{1}{c} J^{\mu} A_{\mu} .
$$

The Lagrangian for the Dirac field $\psi$ is given by $[2,6]$

$$
\mathcal{L}_{\psi}=i \hbar c \bar{\psi} \gamma^{\mu} \partial_{\mu} \psi-m c^{2} \bar{\psi} \psi
$$

While exhibiting global gauge invariance, the Dirac Lagrangian $\mathcal{L}_{\psi}$ is not locally gauge invariant [26]. The usual quantum field theoretic approach is to mandate local gauge symmetry $[3,6]$, thereby requiring subsequent introduction of a new vector field $A_{\mu}$ in order to meet this mandate [2-6]. The current formalism does not require such a method.

The Lagrangian for the coupled field is thus

$$
\begin{aligned}
\mathcal{L}_{\left(\psi, A_{\mu}\right)} \equiv & \mathcal{L}_{\psi}+\mathcal{L}_{A_{\mu}} \\
= & {\left[i \hbar c \bar{\psi} \gamma^{\mu} \partial_{\mu} \psi-m c^{2} \bar{\psi} \psi\right] } \\
& -\frac{1}{16 \pi} H^{\mu \nu} H_{\mu \nu}-\left(e \bar{\psi} \gamma^{\mu} \psi\right) A_{\mu},
\end{aligned}
$$

where $c e \bar{\psi} \gamma^{\mu} \psi=J^{\mu}$ is the quantum field current density satisfying the conservation equation $[2,6,7]$

$$
\partial_{\mu} J^{\mu}=0 .
$$

This is an important result; for the conservation equation is a consequence of the intrinsic

\footnotetext{
${ }^{5}$ Where $A_{\mu}$ is now taken to represent the electromagnetic four-vector potential.

${ }^{6}$ This gauge condition is often incorrectly referred to as the Lorentz condition, vice the correct attribution as the Lorenz condition [23].
}

gauge symmetry of $\mathcal{L}_{\left(\psi, A_{\mu}\right)}$, since $J^{\mu}$ is simply the Noether current corresponding to the local phase transformation $\psi \rightarrow e^{i \alpha(x)} \psi$ concomitant with Eq. (19) as part of the local gauge invariance transformation [21]. As the Ward identity, given by $k_{\mu} \mathcal{M}^{\mu}(k)=0$, is an expression which results from this current conservation, ${ }^{7}$ it follows that the Ward identity is intrinsically manifest as well in the current formalism as a consequence of the inherent local gauge symmetry of the Lagrangian. ${ }^{8}$

The form of the interaction term $\left(e \bar{\psi} \gamma^{\mu} \psi\right) A_{\mu}$ of $\mathcal{L}_{\left(\psi, A_{\mu}\right)}$ arises naturally in this formalism. An intrinsically coupled field must have a coupling parameter - in this case $e$, the electric charge - and a Lagrangian interaction term $[2,3,6]$. Further, in relativistic quantum mechanics, the probability current $\bar{\psi} \gamma^{\mu} \psi$ takes the role of the conserved current $J^{\mu}$ of the wave function $\psi[2,7,21]$. It is natural then to integrate the coupling parameter along with the probability current into the interaction term of Eq. (20). This results in the selfsame interaction term found via the standard derivation through imposed local gauge symmetry $[2,6,21,22]$.

$\mathcal{L}_{\left(\psi, A_{\mu}\right)}$ is locally gauge invariant $[2,3,6,7,22]$. The alternative formalism thus produces a coupled field $\left(\psi, A_{\mu}\right)$ which is represented by an internally local gauge symmetric Lagrangian. There is no need then to either mandate local gauge invariance or thereafter to introduce an external field to meet the mandate, as both are inherent to the formalism; symmetry exists from inception.

Lastly, it is seen that $\mathcal{L}_{\left(\psi, A_{\mu}\right)} \equiv \mathcal{L}_{Q E D}$, the Lagrangian of Quantum Electrodynamics. ${ }^{9}$ In canonically quantizing the theory this equivalence of Lagrangians is conditioned on modification of the

${ }^{7}$ Ref. [21], sections 5.5 and 7.4. Where $\mathcal{M}(k)=$ $\epsilon_{\mu}(k) \mathcal{M}^{\mu}(k)$ is the amplitude for some quantum electrodynamic process involving an external photon with momentum $k$.

${ }^{8}$ Ref. [2], section 13.2.4 (Local gauge invariance $\longleftrightarrow$ current conservation $\longleftrightarrow$ Ward identities).

${ }^{9}$ This paper does not contemplate the Yang-Mills generalization and extension of gauge invariance to non-abelian groups such as $\mathrm{U}(1) \otimes \mathrm{SU}(2)$ of the weak interaction or quantum chromodynamic's SU(3) [21,22], but only a formalism for an intrinsic local U(1) symmetry of QED. Therefore, such symmetries as the Becchi, Rouet, Stora and Tyutin (BRST) symmetry which is typically covered in quantization of nonabelian gauge theories is not addressed herein, but is left to the possible extension of this paper's formalism to such non-abelian generalizations with their associated invariant full effective Lagrangians [22]. 
Papers in Physics, vol. 9, ART. 090002 (2017) / B. Wolk

Lorenz condition relied on above in generating $\mathcal{L}_{A_{\mu}}$. For the canonically quantized formalism, Gupta-Bleuler's weak Lorenz condition given by $\partial_{\mu} A^{\mu+}|\Psi\rangle=0$ replaces the Lorenz condition, in which $A^{\mu+}$ acts as the photon lowering quantum field operator and $|\Psi\rangle$ represents a ket of any number of photons $[2,21,22] .{ }^{10}$ It follows from this conditioned equivalence that the new formalism generates all of electrodynamics and specifies the current produced by the subject Dirac fields $[2,3,6,21] .^{11}$

\section{Conclusion}

Local gauge symmetry plays the central, dominant role in modern field theory [22]. That being the case, it would be preferable that the intrinsic structure of fundamental physical theories exhibit this symmetry $a b$ initio. Therefore, a formalism which produces the Dirac operator equation exhibiting inherent local gauge invariance while also jettisoning the need for invention of an auxiliary vector field in order to satisfy an imposed symmetry constraint is more satisfying from a theoretic standpoint. This paper's formalism achieves such an internal local symmetry, and in doing so naturally generates the fundamental equations of Quantum Electrodynamics. Such a unified description of these basic equations and their processes may also lead to a deeper understanding of the origin of these phenomena.

[1] P A M Dirac, The quantum theory of the electron, Proc. Roy. Soc. Lond. A117, 610 (1928); Ibid, part II, A118, 351 (1928).

[2] R D Klauber, Student friendly quantum field theory, Sandtrove Press, Fairfield, Iowa (2013).

[3] S Weinberg, The quantum theory of fields, Vol. I, Cambridge University Press, Cambridge (2005).

[4] P J E Peebles, Quantum mechanics, Princeton University Press, Princeton, New Jersey (1992).

\footnotetext{
${ }^{10}$ This modification functions as a necessary constraint on the longitudinal and scalar photons in any given quantum state, permitting their mutual cancellation when calculating the Hamiltonian expectation value of the quantized field theory $[2,22]$.

${ }^{11}$ See, e.g., Ref. [6], p. 360 and Ref. [21], p. 78.
}

[5] A Zee, Quantum field theory in a nutshell, Princeton University Press, Princeton, New Jersey (2003).

[6] D Griffiths, Introduction to elementary particles, 2nd Rev. Ed., Wiley-VCH, Weinheim, Germany (2008).

[7] W Pauli, Wave mechanics, Pauli Lectures on Physics, Vol. 5, Dover Publications Inc., Mineola, New York (1973).

[8] R Penrose, W Rindler, Spinors and spacetime, Vol. I, Cambridge University Press, Cambridge (1984); Ibid Vol. II (1986).

[9] P A M Dirac, Directions in physics, John Wiley \& Sons, New York (1978).

[10] D Hestenes, G Sobczyk, Clifford algebra to geometric calculus, Reidel, Dordrecht (1999).

[11] J Dieudonne, Foundations of modern analysis, Academic Press, New York (1960).

[12] O Veblen, J W Young, Projective geometry, Ginn \& Co., Boston (1918).

[13] P Lounesto, Clifford algebras and spinors, Cambridge University Press, Cambridge (2001).

[14] T W Judson, Abstract algebra, PWS Publishing, Texas (1994).

[15] G E Hay, Vector and tensor analysis, Dover Publications Inc. Mineola, New York (1953).

[16] M Schwartz, Principles of electrodynamics, Dover Publications Inc., Mineola, New York (1972).

[17] J R Lucas, P E Hodgson, Spacetime and electromagnetism: An essay on the philosophy of the special theory of relativity, Clarendon Press, New York (1990).

[18] W Pauli, Theory of relativity, Pergamon Press, Oxford (1958).

[19] W Greiner, Classical electrodynamics, Springer-Verlag, New York (1998).

[20] $\mathrm{R}$ Penrose, The road to reality:A complete guide to the laws of the universe, Vintage Books, New York (2004). 
Papers in Physics, vol. 9, ART. 090002 (2017) / B. Wolk

[21] M E Peskin, D V Schroeder, An introduction to quantum field theory, (Economy Edition), Westview Press, Reading, Massachusetts (2016).

[22] G Sterman, An introduction to quantum field theory, Cambridge University Press, Cambridge (1993).

[23] J D Jackson, L B Okun, Historical roots of gauge invariance, Rev. Mod. Phys. 73, 663 (2001). 